\title{
Überraschung im Röntgenbild: Wo ist das Herz geblieben?
}

\begin{abstract}
Ein 32-jähriger Mann, der 20 Jahre stark geraucht hatte, wurde wegen eines nicht produktiven nächtlichen Hustens, Dyspnoe, linksseitigen Thoraxschmerzen und eines unfreiwilligen Gewichtsverlusts von sieben Kilogramm untersucht. Die Symptomatik war etwa vor vier Wochen aufgetreten. Bei der körperlichen Untersuchung bemerkte man eine flache Atmung und sehr leise Herztöne. Vergrößerte Lymphknoten oder sonstige tastbare Raumforderungen wurden nicht festgestellt.
\end{abstract}

— Die Überraschung brachte ein Röntgenbild des Thorax. Fast der gesamte Thoraxraum war durch eine riesige Raumforderung ausgefüllt, ohne dass eine Deviation der Trachea aufgetreten war (Abb. A). Im CT des Thorax mit Kontrastmittel bestätigte sich eine riesige homogene Raumforderung im vorderen Mediastinum ohne Verkalkungen, Fetteinlagerungen und ohne Verlagerung der großen Gefäße (Abb. B). In der koronaren Rekonstruktion des CT wird erkennbar, dass das Herz nach Kaudal verdrängt und teilweise komprimiert wird. Histologisch ergab sich ein Seminom. Der Patient wurde in 15 Sitzungen mit insgesamt $800 \mathrm{cGy}$ bestrahlt und erhielt sechs Zyklen mit Cisplatin und Etoposid. Darunter bildeten sich die Symptomatik und der Tumor rasch zurück.

\section{Kommentar}

Bei soliden Primärtumoren des vorderen Mediastinums ist an retrostenale Strumen, Thymome, Teratome oder primäre Lymphknotenerkrankungen zu denken. Selten sind Perikardtumoren, Paragangliome, Lipome und Hämangiome. Bei zystischen Prozessen kommen Thymuszysten, Teratome, Dermoidzysten oder auch Strumen in Betracht. Diese Tumoren weisen häufig Kalzifikationen auf. Die häufigsten Tumoren des vorderen Mediastinums sind im mittleren Lebensalter Thymome. Unter
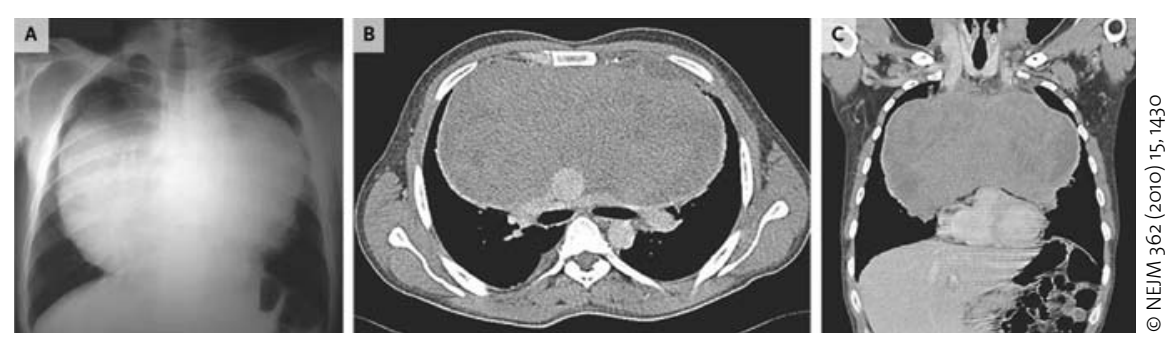

Die Raumforderung im Röntgen-Thorax und im CT.

den mediastinalen Keimzelltumoren ist das Seminom am häufigsten. Testikuläre maligne Keimzelltumoren machen zwar nur $1 \%$ aller Neoplasien aus, gehören jedoch mit 23\% der bösartigen Neubildungen zu den häufigsten Tumorleiden bei Männern bis zum Alter von 45 Jahren. Die Inzidenz ist in den letzten Jahrzehnten erheblich angestiegen. Gleichzeitig hat sich die Prognose dank guter therapeutischer Möglichkeiten verbessert. Heute werden mehr als $80 \%$ der Patienten unabhängig vom initialen Tumorstadium geheilt.

H. S. FÜEßL =

- J. C. Romo, I. Hernandez

(Hospital Angeles del Pedregal, Mexico City, Mexico): Mediastinal mass. New Engl. J. Med. $362(2010) 15,1430$

\section{Homöopathie für Senioren}

Kann man typische Altersleiden auch mit Homöopathika ganz gut in den Griff bekommmen?

- Deutsche Forscher beobachteten zwei Jahre lang 83 Senioren (> 70 Jahre), die homöopathische Ärzte konsultiert hatten. Das Ziel der Studie war, die häufigsten Erkrankungen und deren Verlauf zu ermitteln.

Die Auswertungen ergeben, dass am häufigsten eine Hypertonie vorlag. Der Schweregrad der Symptome nahm im Verlauf der Therapie signifikant ab. Die Lebensqualität blieb dagegen unverändert. Die Autoren ziehen aus diesen Daten den Schluss, dass eine homöopathische Therapie bei diesen Patienten angebracht sein könnte, und dass Äquivalenzstudien zur Überprüfung dieser Hypothese nötig seien.

\section{Kommentar}

Es handelt sich hier um eine Sekundäranalyse einer bereits mehrfach publizierten Beobachtungsstudie. Ich fürchte, dass auch diese vierte Publikation die nunmehr zehn Jahre alten Daten nicht besser macht. Falls mich jemand davon überzeugen will, dass Homöopathie bei der Therapie der Hypertonie wirksam ist, rate ich, mir Blutdruckdaten zu liefern. Dergleichen findet sich jedoch nicht in dieser Arbeit. Der Parameter „Schweregrad der Symptome" sagt wenig aus (insbesondere in Bezug auf Hypertonie). Kausalzusammenhänge können aus dieser Studie jedenfalls keine abgeleitet werden. Auch die Schlussfolgerung, dass Äquivalenzstudien nötig seien, erscheint mir verwunderlich. Wenn die hier vorgelegten Daten es überhaupt wert sind, weiter verfolgt zu werden, dann sollten placebokontrollierte Studien prüfen, $o b$ Homöopathika die bei dieser Zielgruppe häufigste Erkrankung (Hypertonie) positiv beeinflussen.

\section{E. ERNST =}

\section{- M. Teut et al.}

Homeopathic treatment of elderly patients - a prospective observational study with follow-up over a two year period. BMC Geriatr. $22(2010) 10$ 